\title{
Ordered droplet structures at the liquid crystal surface and elastic-capillary colloidal interactions
}

\author{
I.I. Smalyukh ${ }^{1}$, S. Chernyshuk ${ }^{2}$, B.I. $\mathrm{Lev}^{2,3}$, A.B. $\mathrm{Nych}^{2}$, U. Ognysta ${ }^{2}$, V.G. Nazarenkoº \\ and O.D. Lavrentovich ${ }^{1}(*)$ \\ ${ }^{1}$ Liquid Crystal Institute and Chemical Physics \\ Interdisciplinary Program, Kent State University, Kent, OH 44242, USA \\ ${ }^{2}$ Institute of Physics, Prospect Nauki 46, Kyiv-39, 03039, Ukraine \\ ${ }^{3}$ Japan Science and Technology Corporation, Yokoyama Nano-structured \\ Liquid Crystal Project, 5-9-9 Tokodai, Ibaraki 300-2635, Japan
}

(July 6, 2018)

\begin{abstract}
We demonstrate a variety of ordered patterns, including hexagonal structures and chains, formed by colloidal particles (droplets) at the free surface of a nematic liquid crystal (LC). The surface placement introduces a new type of particle interaction as compared to particles entirely in the LC bulk. Namely, director deformations caused by the particles lead to distortions of the interface and thus to capillary attraction. The elastic-capillary coupling is strong enough to remain relevant even at the micron scale when its buoyancycapillary counterpart becomes irrelevant.
\end{abstract}

Typeset using REVTEX

*Author for correspondence (e-mail: odl@lci.kent.edu) 
2D organization of nanometer- and micrometer-sized colloidal particles at fluid interfaces is a fascinating phenomenon of both fundamental and applied interest. The nature of micron-scale interparticle forces, especially of the attractive nature, remains a subject of an ongoing debate [1-9]. In most cases, the interparticle forces are isotropic and cause hexagonal ordering. Anisotropic interactions can be achieved when the particles are nonspherical [4,5]. Apparently, the surfaces of anisotropic fluids, i.e., liquid crystals (LCs), can also support anisotropic interactions and thus a richer variety of ordered patterns as compared to isotropic fluids, but the current knowledge in the field is rather limited. It is known that small particles might form chains decorating the surface director field $[10,11]$ and that the surface air bubbles can be accompanied by point defects [12]. The behavior of particles entirely in the LC bulk is studied much better [13-21]. Because of the anisotropy of molecular interactions at the particle surface (the phenomenon of anchoring [22]), the embedded particle causes director distortions. The most frequently met distortions are of dipole symmetry; they lead to chaining of droplets [13]. Recently, an unexpected and so far unexplained hexagonal structures have been discovered for an array of glycerol droplets assumed to be in the nematic bulk [18-20] and for droplets in smectic membranes [21].

In this work, we use confocal microscopy to demonstrate that placement of colloidal particles (glycerol droplets of radius $R=1-10 \mu \mathrm{m}$ ) at the $L C$ surface leads to attractive interactions and ordered patterns of hexagonal and chain type that depend on the thickness of the LC film. The attractive interactions can be explained by the elastic-capillary coupling, as the particle-induced director deformations distort the LC surface.

The glycerol droplets are obtained as in Ref. [18]. A Petri dish containing a layer of glycerol and the nematic LC pentylcyanobiphenyl (5CB, EM Industries) on top of it, is kept at $50^{\circ} \mathrm{C}$ for $10 \mathrm{~min}$, to facilitate diffusion of the glycerol molecules into the $5 \mathrm{CB}$ layer which is in the isotropic phase above $\approx 35^{\circ} \mathrm{C}$. When the sample is cooled down, solubility decreases and one observes appearance and growth of glycerol droplets. The technique produces droplets of a constant radius $R$ determined by the cooling rate and the number of thermal cyclings. The thickness of the LC film is controlled in the range $h=(3-100) \mu m$. 
The 5CB film is in the hybrid aligned state: the director $\hat{\mathbf{n}}$ is parallel to the LC-glycerol interface at the bottom and perpendicular to the air-LC interface at the top.

In order to determine the location of droplets, we use the fluorescence confocal polarizing microscopy (FCPM) [23]. Two different dyes with separated absorption and fluorescence bands, fluorescein and Nile red (Aldrich), were added (0.01 wt \%) to tag glycerol and LC, respectively [23]; they did not change the appearance of patterns. The FCPM textures of vertical cross-sections, Fig.1a-c, unambiguously demonstrate that the glycerol droplets are trapped at the LC-air interface. For a better clarity, the images in Fig.1a,b are taken for a large drop; smaller drops are also located at the interface, Fig.1c.

A 4-6 hour relaxation at room temperature results in 2D ordered structures of droplets. The order is hexagonal when the LC layer is thick, $h>>R, h=(20-100) \mu m$, Fig.2a,b. The droplet center-to-center separation $r_{e q}$ increases with the droplets diameter 2R, Fig.2d; each data point is an average of the data collected by video-recording the patterns every $6 \mathrm{~s}$ for $10 \mathrm{~min}$. The angles between the directions to the nearest neighbours changes in the range $60^{0} \pm 8^{0}$ for well-equilibrated structures. At this stage, it is hard to quantify the lattice symmetry more precisely as it might be influenced by slowly changing director pattern in the bulk. In thin films, $h / R \approx 2 \div 5,3 \mu m \leq h \leq 10 \mu m$, Fig.3a, the pattern changes completely, as the droplets form chains oriented along the average horizontal projection of $\hat{\mathbf{n}}$. The length of chains (number of droplets in a chain) increases when $h$ decreases. Importantly, both hexagonal and chain patterns disorganize when $5 \mathrm{CB}$ is heated into the isotropic phase.

The very existence of $2 \mathrm{D}$ hexagonal pattern in thick films does not discriminate between attractive and repulsive interactions among the particles, as in the confined systems such as a Petri dish the ordering can be induced by purely repulsive forces. The presence of repulsive force is manifested clearly, as the droplets are well separated, $r_{e q} \approx(3-4) R$, Fig.2d. The following three experiments show that the interaction has also an attractive component. (A) We start with the Petri dish in which the 2D lattice occupies the whole area and then use a filter paper to wipe about $1 / 2-1 / 3$ of the droplets. The remaining droplets restore the $2 \mathrm{D}$ lattice within the smaller area, with the lattice parameter close to 
the original one. (B) We change the direction of the meniscus tilt near the Petri dish walls by coating the walls with a hydrophobic material (stearin); the types of the 2D ordering remain the same. (C) After the 2D lattice is formed in a regular manner, we add (through a microsyringe) a small amount ( $0.01 \%$ by weight) of a surfactant (cetylpiridinium chloride) to the nematic layer. The interparticle distances decrease; the shrinked clusters are separated by droplet-free areas, Fig.2c.

What is the mechanism of the droplet attraction? The gravity-mediated capillary effect and the van der Waals forces appear to be insignificant, as the corresponding pair potentials $[24,22]$

$$
U_{g}(r) \approx \frac{2 \pi R^{6}}{\sigma_{L C A}} g^{2} \rho_{L C}^{2} \ln \left(\frac{r}{\lambda}\right) ; \quad U_{v d W}(r) \approx-\frac{A R}{12(r-2 R)}
$$

are of the order of thermal energy $k_{B} T \approx 4 \times 10^{-21} J$ or less, when $r$ is a few microns. Here $\sigma_{L C A} \approx 3.8 * 10^{-2} \mathrm{~J} / \mathrm{m}^{2}[25]$ is the LC-air surface tension coefficient, $\rho_{L C} \approx 10^{3} \mathrm{~kg} / \mathrm{m}^{3}$ is the LC density, $g$ is acceleration due to the gravity, $\lambda=\sqrt{\sigma_{L C A} /\left(g \rho_{L C}-\frac{\partial \Pi}{\partial h}\right)}$ is the capillary length; $\Pi \approx K / h^{2}$ is the elastic disjoining pressure for the hybrid aligned film [22]; $K \sim 10^{-11} N$ is the average nematic elastic constant; in all cases of interest to us, $\lambda>>r_{e q}$, as $\lambda \approx 2 m m$ when $h \rightarrow \infty$ and $\lambda \approx 0.5 m m$ when $h=5 \mu m$; finally, $A$ is the Hamaker constant of the order of $10^{-19} \mathrm{~J}$ or smaller [22].

Attraction of droplets at the nematic surface (and absence of attraction and ordering when $5 \mathrm{CB}$ is in the isotropic state) can be explained by the coupling of orientational elasticity and capillarity. The elastic distortions caused by the submerged part of the particle give rise to a vertically resolved force $f_{e l}$ that shifts the LC-air interface upward or downward, depending on the particle properties, to reduce the elastic energy of the LC host; $f_{e l}$ is balanced by surface tension. Non-flat interface causes long-range attraction of particles. As the first approximation, we divide the total interaction potential $U(r)$ into two parts, $U_{\text {elc }}(r)$ describing the elastic-capillary coupling and $U_{\text {elb }}(r)$ describing the "pure bulk" elastic interactions.

To estimate the orders of magnitude, we use the idea that $f_{e l}$ is related to the 
non-vanishing surface anchoring of $\hat{\mathbf{n}}(r)$ at the LC-particle interface: $f_{e l}=\frac{\partial}{\partial z} E_{e l}(z) \sim$ $\frac{\partial}{\partial z} \oint_{\Omega(z)} W(\hat{\mathbf{n}} \cdot \hat{\nu})^{2} d s$, where $E_{e l}(z)$ is the total elastic energy of distortions caused by the particle, $\Omega(z)$ is part of the droplet surface immersed in the LC, $W$ is the (polar) anchoring coefficient of the LC-glycerol interface, $\hat{\nu}$ is the normal to the interface, and $z$ is the vertical coordinate of some fixed point, say, the bottom of the droplet. For $f_{e l} \approx 4 \pi W R$, with $W=10^{-5} \mathrm{~J} / \mathrm{m}^{2}[22]$, one finds $f_{e l} \sim 10^{-10} N \sim 10 \mathrm{~K}$ when $R=1 \mu \mathrm{m}$. The force $f_{e l}$ replaces the buoyancy force in the standard treatment [3,24]; it is balanced by the surface tension, hence, $f_{e l}=2 \pi \sigma_{L C A} r_{i} \sin \psi$, where $\psi$ is the meniscus slope at the triple contact line of radius $r_{i}$. The vertical displacement $\xi(r) \approx-r_{i} \sin \psi \ln \frac{r}{\lambda}$ changes the interfacial area and leads to the attractive potential written for two identical particles as $U_{e l c}=-f_{e l} \xi$, or

$$
U_{\text {elc }}(r)=\frac{f_{e l}^{2}}{2 \pi \sigma_{L C A}} \ln \left(\frac{r}{\lambda}\right),
$$

similar to the buoyancy case [3,24]. For $f_{\text {el }} \sim 10 \mathrm{~K}$, one estimates $U_{\text {elc }} \sim 3 \times 10^{-19} \mathrm{~J} \sim 80$ $k_{B} T$. The elastic-capillary coupling is strong enough to keep droplets attracted even at micron scales where the gravity mechanism vanishes: the dependence $U_{\text {elc }}(R)$ is weaker than $U_{g}(R) \propto R^{6}$ in Eq.(1), as $E_{e l}(R) \sim W R^{2}$ for $R<<K / W$, and $E_{e l}(R) \sim K R$ for $R>>K / W[22]$.

We turn now to the "pure bulk" elastic interactions. In the hybrid aligned film, $\hat{\mathbf{n}}$ is determined by the balance of anchoring and elasticity. In the thick films $(h>>K / W$, $h>>R$ ), $\hat{\mathbf{n}}$ at the LC-air interface is vertical. Tangential anchoring of $\hat{\mathbf{n}}$ at the droplet surface leads to a point defect-boojum [22] close to the south pole, Fig.1e and 2e. The distortions $\hat{\mathbf{n}}(\mathbf{r})$ around each droplet can be approximated by an elastic dipole normal to the LC-air interface [13], $\mathbf{p} \approx\left(0,0, \alpha_{z} R^{2}\right)$, where $\alpha_{z}$ is a constant. The bulk elastic dipoledipole interaction is thus isotropic in the $x y$ plane and repulsive,

$$
U_{\text {elb,thick }}(r)=K \frac{\alpha_{z}^{2} R^{4}}{r^{3}} .
$$

The total pair potential, $U=U_{e l c}+U_{e l b, t h i c k}$, has a clear minimum at

$$
r_{e q}^{p a i r}=\left(\frac{6 \pi \alpha_{z}^{2} \sigma_{L C A} R^{4} K}{f_{e l}^{2}}\right)^{1 / 3}
$$


e.g., $r_{e q}^{\text {pair }}=13 \mu m$ for $f_{e l}=10^{-10} N, \alpha_{z}=0.2$ and $R=3 \mu m$. For $f_{e l} \propto W R$, Eq.(4) predicts $r_{e q}^{\text {pair }}=\gamma\left(\sigma_{L C A} R^{2} K / W^{2}\right)^{1 / 3} \propto R^{2 / 3}$, where $\gamma$ is a dimensionless constant. The trend is in agreement with the experiment, Fig.2d, despite the fact that Eq.(4) is derived from the pair potential. Generally, $f_{e l}$ might be a non-linear function of $R$ and also might depend on $r$. A surfactant can be used to control the patterns, as it changes $\sigma_{L C A}$, anchoring at interfaces, and thus $f_{e l}$. Sometimes, one observes the patches of a hexagonal "dense" pattern with $r_{e q, d} \approx(2.2-2.7) R$ that coexist with the regular hexagonal patterns of a larger period $r_{e q} \approx(3-4) R$ (but the same $R$ ). These dense patches are not understood yet, and might be provoked by ionic and flexoelectric charge, by the $r$-dependence of $f_{e l}$, surfactant contamination, etc.

In the thin films, $\hat{\mathbf{n}}$ becomes progressively horizontal at the LC-air interface as the anchoring coefficient $W_{\text {air }}$ there is smaller than $W[26]$. When $h$ decreases to $2 R$ and $K / W_{\text {air }}$, the boojum shifts to the side, Fig.3b, giving rise to the in-plane elastic dipole component, $p_{x}=\alpha_{x} R^{2}$, and an anisotropic interaction contribution

$$
U_{\text {elb }, \text { thin }}(r, \theta)=K \frac{\alpha_{x}^{2} R^{4}}{r^{3}}\left(1-3 \cos ^{2} \theta\right),
$$

that depends on the angle $\theta$ between the radius-vector connecting the two droplets and the direction of $p_{x}$ 's. The chain formation can thus be explained similarly to the chaining of droplet-hedgehog pairs entirely in the LC bulk [13]: $U_{\text {elb,thin }}$ is minimum when $\theta=0$. The analogy is not complete, as our problem is of a lower symmetry and requires one to consider $h^{-}, W_{a i r^{-}}$, and $W$-dependences of $\hat{\mathbf{n}}(\mathbf{r})$, etc. For example, if the film is so thin that $\hat{\mathbf{n}}$ is parallel to the LC-air interface [26], then $\hat{\mathbf{n}}(\mathbf{r})$ around the droplets might become of a quadrupolar type with two boojums along the horizontal axis.

To conclude, the particles (glycerol droplets) at the LC-air interface are capable of both repulsive and attractive interactions that lead to ordered patterns of hexagonal and chain types. The director distortions in LC give rise to a vertical elastic force balanced by surface tension; the deviation of the LC-air interface from the horizontal plane results in attraction of particles. The phenomenon is general, as we observe hexagonal patterns not only for the 
liquid droplets but also for micron-size polymer spheres at the nematic-air interface; these results will be published elsewhere. The elastic-capillary mechanism can also shed some light at the observations of hexagonal patterns claimed to form in the bulk of the hybrid aligned films [18-20]. If the particles were indeed in the bulk, where $\hat{\mathbf{n}}$ is tilted, then the elastic interaction potential would be anisotropic and thus generally inconsistent with the hexagonal order. The elastic model [18] does not consider the angular dependence of the potential for tilted $\hat{\mathbf{n}}$ and does not explain the hexagonal ordering. A pure elastic model would not be satisfactory even for thick films, in which $\hat{\mathbf{n}}$ around the droplets is vertical, as in this case the interaction would be only repulsive, Eq.(3). Note that the experiments [18-20] relied on the standard (non-confocal) microscopy with a poor $z$-axis resolution. The particles might have been located at the interface rather than in the LC bulk, which would bring the observations [18-20] into the category of elastic-capillary phenomenon proposed in this work.

We acknowledge helpful discussion with M. Kleman, V. Pergamenshchik, S.V. Shiyanovskii, P. Tomchuk, and H. Yokoyama. The work was supported by National Science Foundation Grant DMR-0315523 and STCU grant \#2025. ODL thanks the participants of the "Geometry and Materials Sciences" Program at the Aspen Center for Physics for discussions. 


\section{Figure captions.}

FIG.1. Two-channel FCPM textures of the vertical cross-section of the LC film: $(a, b)$ a single glycerol droplet at the LC-air interface; (c) a raw of droplets that is a part of a hexagonal pattern at the LC-air interface; (d) scheme of forces and interfacial tension vectors at the LC-air interface with a strongly exaggerated meniscus slope; (e) 3D director field in the LC layer. The LC layer (glycerol droplet) is manifested by the high (low) intensity of fluorescent light in parts $(\mathrm{a}, \mathrm{c})$; the contrast is opposite in (b).

FIG.2. Optical microscopy pictures of hexagonal structures at the free surface of the thick nematic layer, $h \approx 60 \mu m$, formed by droplets of different average diameter: (a) $2 R \approx$ $7 \mu m,(\mathrm{~b}) 2 R \approx 1 \mu m$. (c); arrangement of droplets after the surfactant is added to the nematic layer; (d) droplets separation $r_{e q}$ vs. $2 R$; the data are compared to the dependence $r_{e q}^{\text {pair }}=\gamma\left(\sigma_{L C A} R^{2} K / W^{2}\right)^{1 / 3}$, where $\gamma=0.43, K=10^{-11} \mathrm{~N}, \sigma_{L C A}=3.8 \times 10^{-2} \mathrm{~J} / \mathrm{m}^{2}$, $W=10^{-5} \mathrm{~J} / \mathrm{m}^{2}$, see the text; (e) schematic drawing of the director field in the top part of the LC layer.

FIG.3. Optical microscopy textures of glycerol droplets forming chains at the free surface of a thin LC layer, $h \approx(7-10) \mu m$ (a) and the corresponding director field scheme (b). 


\section{REFERENCES}

[1] P. Pieranski, Contemp. Phys. 24, 25 (1983).

[2] R.E. Kusner, J.A. Mann, J. Kerins, and A.J. Dahm, Phys. Rev. Lett. 73, 3113 (1994).

[3] P. A. Kralchevsky and K. Nagayama, Advances in Colloidal and Interface Science, 85, 145 (2000)

[4] A.B.D. Brown, C.G. Smith, and A.R. Rennie, Phys. Rev. E 62, 951 (2000)

[5] D. Stamou, C. Duschl, D. Johannsmann, Phys. Rev. E 62, 5263 (2000)

[6] K. Zahn, A. Wille, G. Maret, S. Sengupta, and P. Nielaba, Phys. Rev. Lett. 90, 155506 $(2003)$

[7] M.G. Nikolaides, A.R. Bausch, M.F. Hsu, A.D. Dinsmore, M.P. Brenner, C. Gay, and D.A. Weitz, Nature 420, 299-301 (2002) and 424, 1014 (2003).

[8] M. Megens and J. Aizenberg, Nature 424, 1014 (2003).

[9] Y. Han and D.G. Grier, Phys. Rev. Lett. 91, 038302 (2003).

[10] J. Rault, C.R. Acad. Sci. Ser.B 272, 1275 (1971).

[11] P. Cladis, M. Kleman, and P. Pieranki, C.R. Acad. Sci. Ser.B 273, 275 (1971).

[12] R.B. Meyer, Mol. Cryst. Liq. Cryst. 16, 355 (1972).

[13] P. Poulin, H . Stark, T.C. Lubensky, and D.A. Weitz, Science 275, 1770 (1997).

[14] B. I. Lev and P. M. Tomchuk, Phys. Rev. E 59, 591 (1999).

[15] A. Borstnik, H. Stark and S. Žumer, Phys. Rev. E 60, 4210 (1999).

[16] J.-Ch. Loudet, P. Barois, and P. Poulin, Nature 407, 611 (2000).

[17] S. P. Meeker,W. C. K. Poon, J. Crain, and E. M. Terentjev, Phys. Rev. E 61, R6083 $(2000)$. 
[18] V. G. Nazarenko, A. B. Nych, and B. I. Lev, Phys. Rev. Lett. 87, 075504 (2001).

[19] B. Lev, A. Nych, U. Ognysta, D. Reznikov, S. Chernyshuk, and V. Nazarenko, Pis'ma Zh.Exp. Teor. Fiz. 75, 393 (2002) [Sov. Phys. JETP Lett. 75, 322 (2002)].

[20] T. Yamamoto, J. Yamamoto, B.I. Lev, and H. Yokoyama, Appl. Phys. Lett. 81, 2187 (2002).

[21] P. V. Dolganov, E.I. Demikhov, V.K. Dolganov, B.M. Bolotin, and K. Krohn, Eur.Phys. J. E 12, 593 (2003).

[22] M. Kleman and O.D. Lavrentovich, Soft Matter Physics: An Introduction (SpringerVerlag, New York, 2003).

[23] I.I. Smalyukh, S.V. Shiyanovskii, and O.D. Lavrentovich, Chem. Phys. Lett. 336, 88 (2001).

[24] D. Y. C. Chan, J. D. Henry, and L. R. White, J. Colloid Interface Sci. 79, 410 (1981).

[25] M. Tintaru, R. Moldovan, T. Beica, and S. Frunza, Liq. Cryst. 28, 793 (2001).

[26] O.D. Lavrentovich and V.M. Pergamenshchik, Phys. Rev. Lett. 73, 979 (1994). 

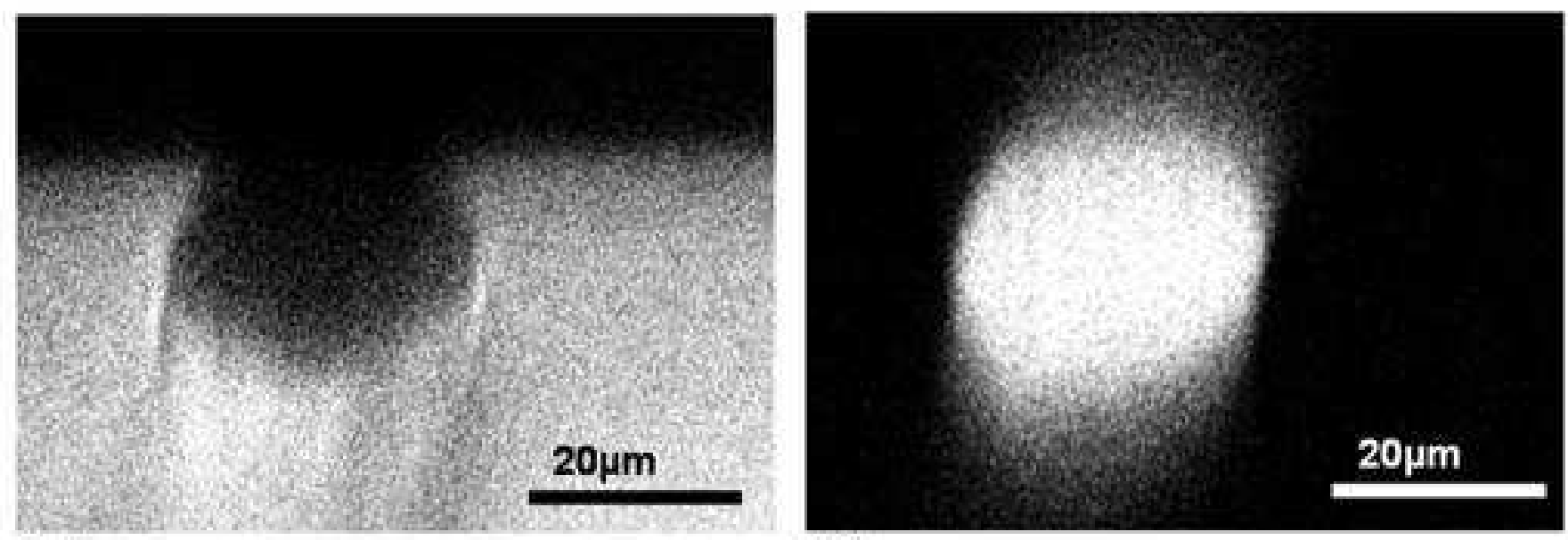

(a)

(b)

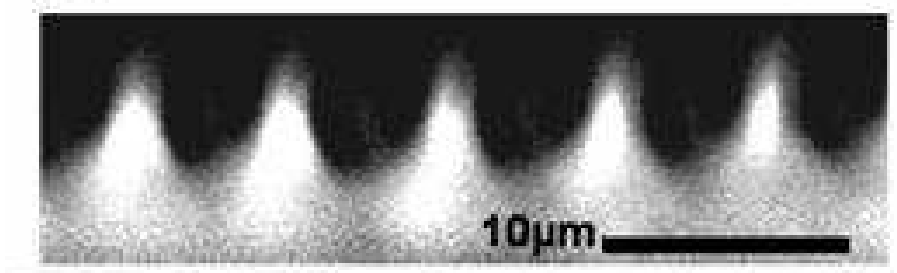

(c)
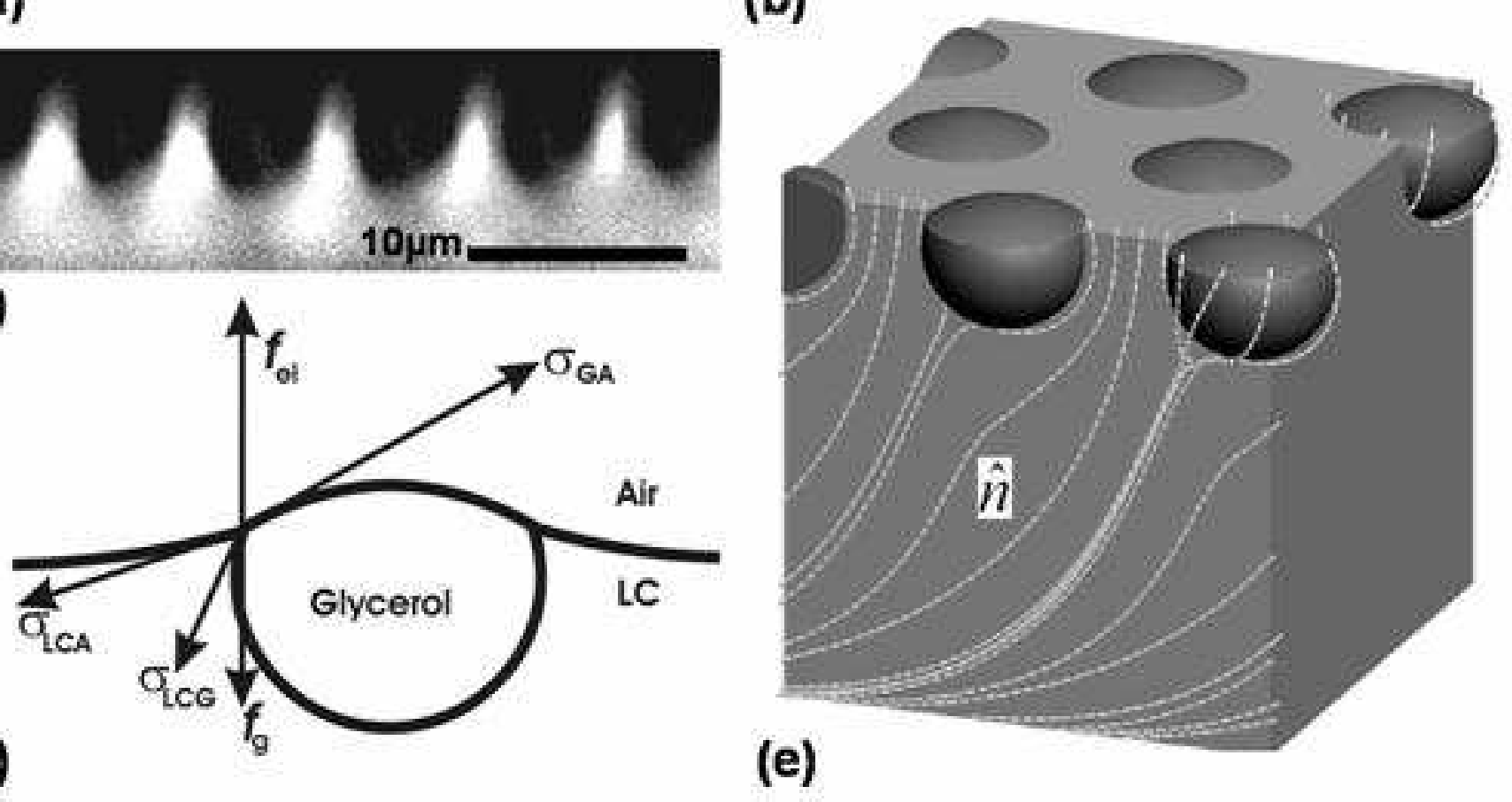

FIG.1 

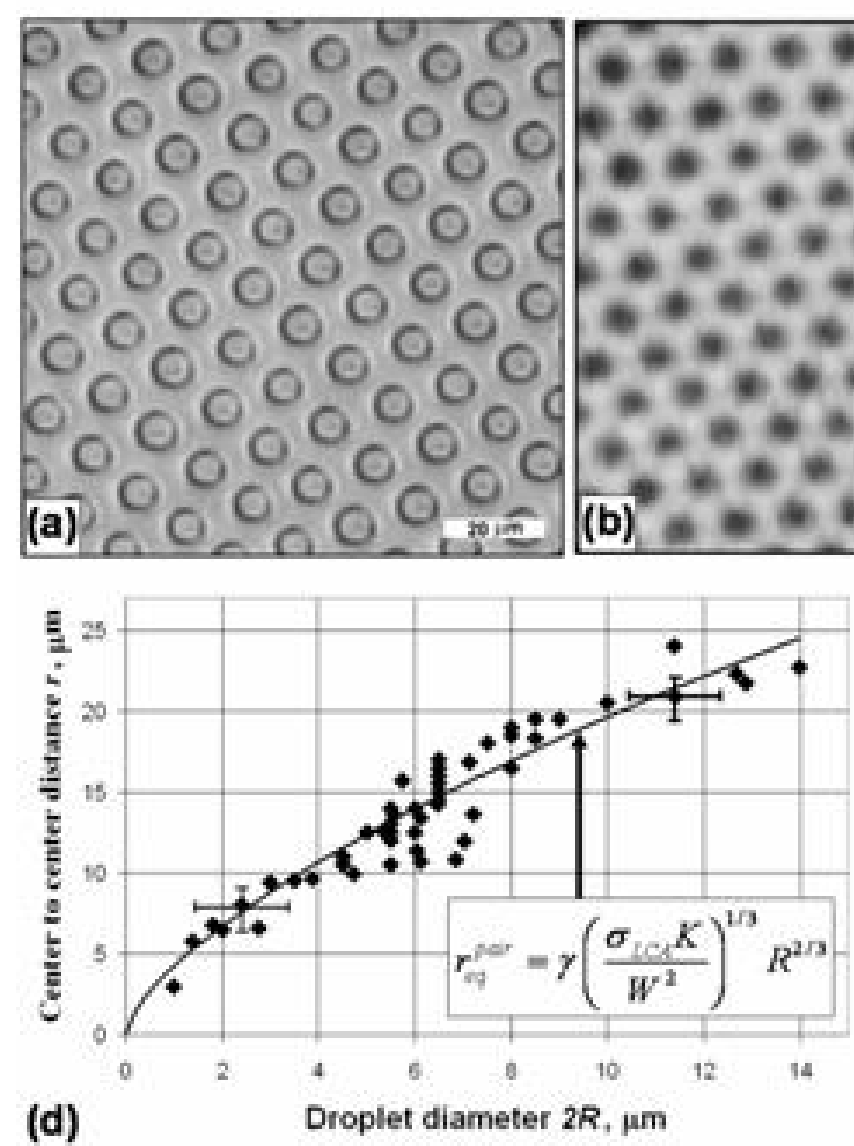
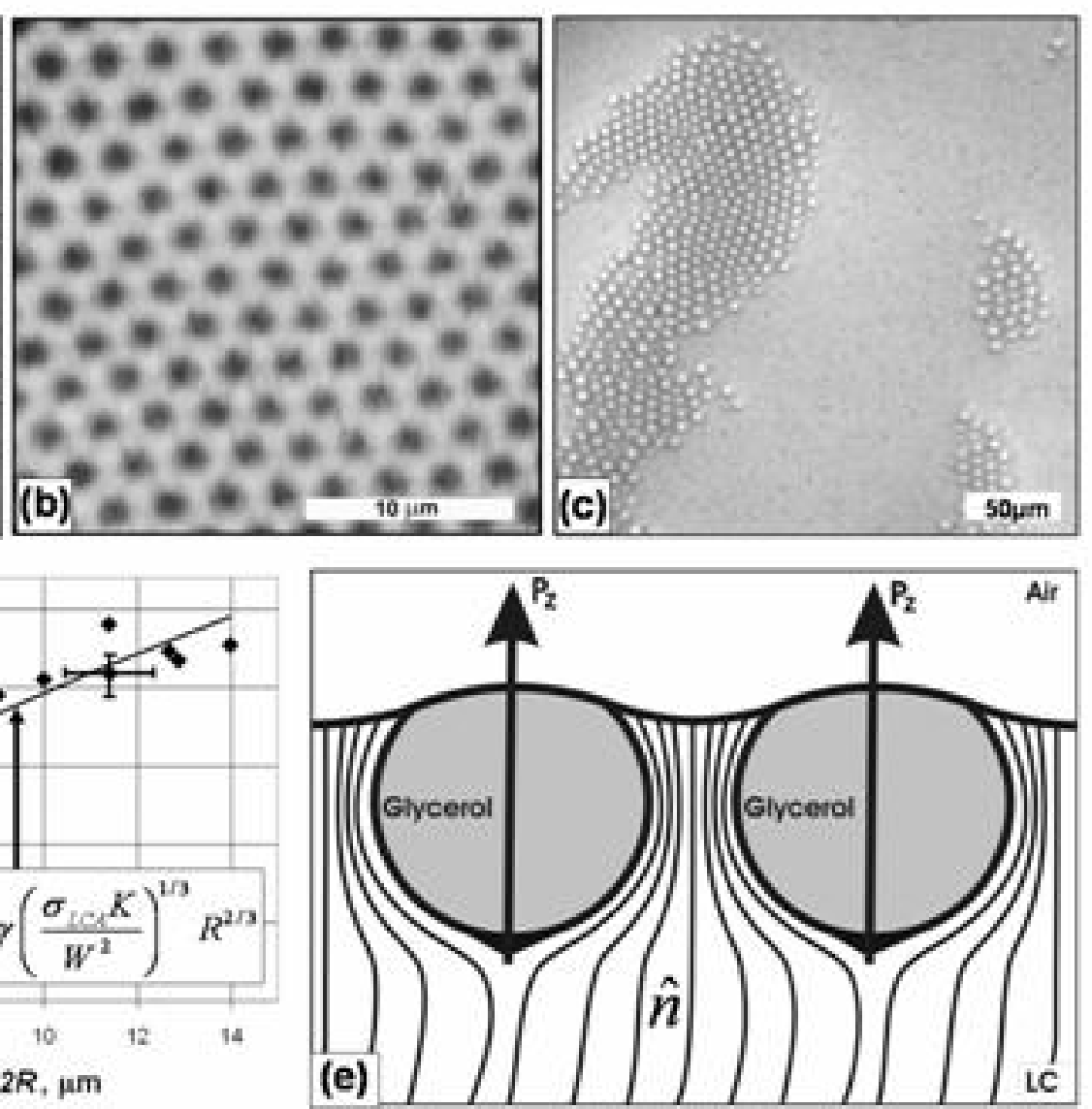

FIG.2 


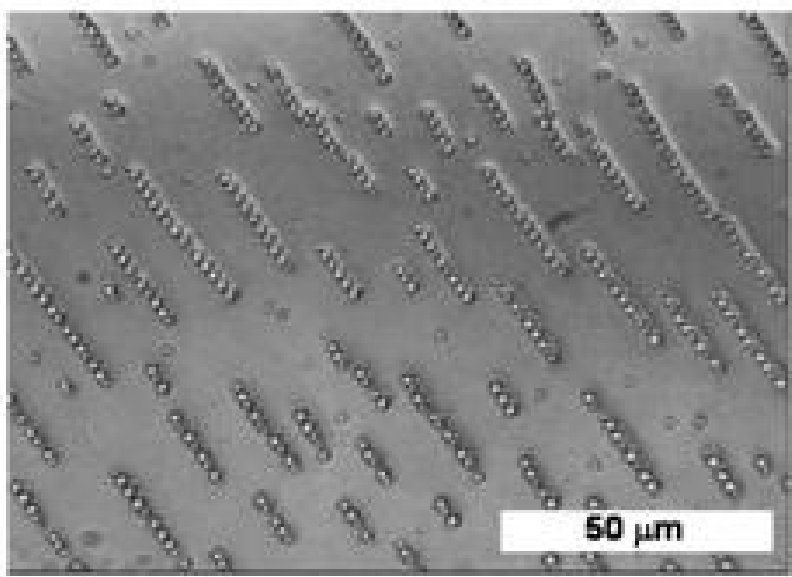

(a)

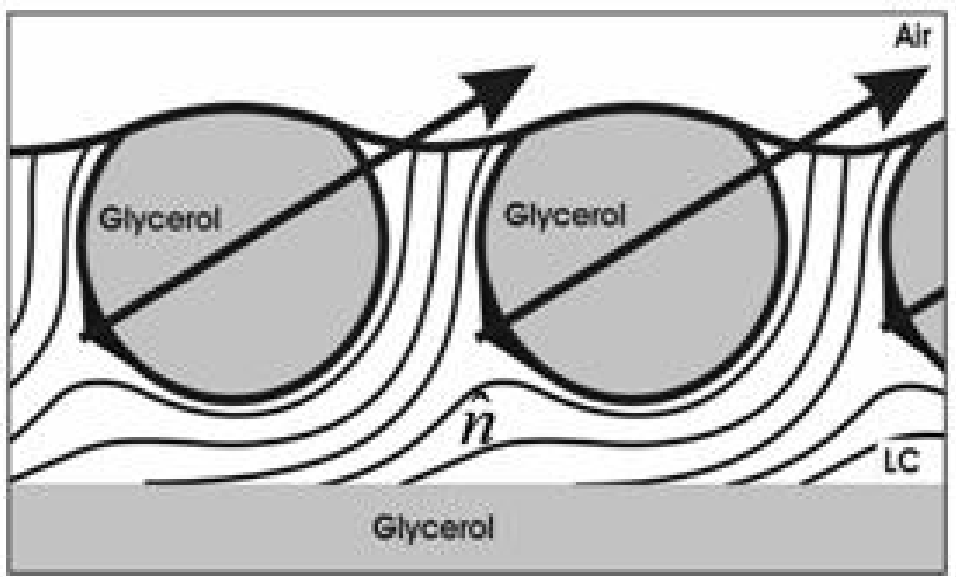

(b)

FIG.3 